\title{
Verification of Low Risk for Perihippocampal Recurrence in Patients with Brain Metastases Who Received Whole-Brain Radiotherapy with Hippocampal Avoidance
}

\section{Youngkyong Kim, $\mathrm{MD}^{1}$ \\ Sung Hwan Kim, MD2 \\ Jong Hoon Lee, MD2 \\ Dae Gyu Kang, PhD²}

${ }^{1}$ Department of Radiation Oncology, Kyung Hee University Hospital, Seoul, ${ }^{2}$ Department of Radiation Oncology, St. Vincent's Hospital, College of Medicine, The Catholic University of Korea, Suwon, Korea
Correspondence: Sung Hwan Kim, MD Department of Radiation Oncology, St. Vincent's Hospital, College of Medicine, The Catholic University of Korea,

93 Jungbu-daero, Paldal-gu, Suwon 16247, Korea Tel: $82-31-249-7560$

Fax: 82-31-257-3734

E-mail: kimandre@catholic.ac.kr

\section{Purpose}

The purpose of this study was to analyze the patterns of failure and survival outcome in patients with brain metastases who received whole-brain radiotherapy (WBRT) with hippocampal avoidance (HA) using simultaneous integrated boost (SIB) on metastatic brain tumors.

\section{Materials and Methods}

We retrospectively reviewed 42 patients treated with HA-WBRT for brain metastases. A total of 25 Gy for whole brain and 35-55 Gy for gross tumors were delivered with 10 fractionations. Local tumor and intracranial progression were defined as a recurrence or tumor progression in SIB field and any recurrence or tumor progression within whole brain, respectively. Progression in HA zone was defined as the recurrence within the area expanded $5 \mathrm{~mm}$ from HA zone.

\section{Results}

Median follow-up duration was 10.0 months (range, 4.1 to 56.4 months). Intracranial progression was observed in 13 patients (31.0\%) and the median duration from the start of HA-WBRT to progression was 10.6 months (range, 0.9 to 33.0 months). Local tumor progression and new metastasis outside SIB field occurred in 10 patients (23.8\%) and nine patients (21.4\%), respectively. There was no isolated hippocampal metastasis, except only one patient (2.4\%) with multiple metastases inside and outside HA zone simultaneously. Median survival time and intracranial progression-free survival rate at 1 year were 19.4 months (95\% confidence interval [Cl], 9.6 to 29.2$)$ and $71.5 \%$, respectively, and those for overall survival were 26.5 months $(95 \% \mathrm{Cl}, 15.4$ to 37.5$)$ and $67.9 \%$, respectively.

\section{Conclusion}

HA-WBRT was associated with low risk of new metastasis in HA region in the patients with brain metastases. These findings would serve as useful guidance on applying HA-WBRT in clinical practice.

\section{Introduction}

Brain metastases are common manifestation of advanced cancers, observed in up to $30 \%$ of cancer patients [1]. Although whole brain radiotherapy (WBRT) increases the survival up to 3-6 months, poor prognosis is still predicted for the patients with brain metastasis [2]. WBRT had been
Key words

Brain, Neoplasm metastasis, Hippocampus, Radiotherapy, Recurrence 
within the hippocampus. These neural progenitor cells seemed to be more apoptotic and gliogenic after cranial irradiation [10-12]. Based on the hippocampal contribution to maintaining neurocognitive function, and the development of sophisticated radiation delivery technique, a phase II trial of Radiation Therapy Oncology Group (RTOG) 0933 was proposed to verify the hypothesis that hippocampal sparing during WBRT for the patients with brain metastases might reduce or delay the incidence and/or severity of neurocognitive function decline as well as control the intracranial disease. Safety of RTOG 0933 trial was based on the reliable estimation that the risk of disease progression within the hippocampal avoidance (HA) region was not significant [13].

Few studies have so far reported the incidences and risk of new metastasis near the hippocampus in the patients treated with HA-WBRT for multiple brain metastases. Against this background, the current study was designed to analyze the treatment outcome and patterns of failure in the patients with brain metastases who received HA-WBRT.

\section{Materials and Methods}

\section{Patients and radiation treatment}

Between September 2011 and October 2017, HA-WBRT was implemented in 65 patients with brain metastases at St. Vincent's Hospital in the Republic of Korea. Of them, 23 patients were excluded from the analysis for the following reasons; 14 patients for the absence of follow-up magnetic resonance imaging (MRI), six for the follow-up duration of less than 4 months, three for incomplete treatment. The clinical data included the age at WBRT, performance status, the site and histology of primary origin, status of extracranial disease, the number and size of metastatic tumors in brain, and radiotherapy (RT) technique, dose, and duration.

All patients received HA-WBRT with simultaneous integrated boost (SIB) on gross tumors. A total of $25 \mathrm{~Gy}$ for whole brain and 35-55 Gy for gross tumors were delivered with 10 fractionations by tomotherapy (TomoTherapy Incorporated, Madison, WI). The converted dose with radiobiologically weighted dose equivalent of 2 Gy per fraction (EQD2), calculated using a linear quadratic model with a $\alpha / \beta$ ratio of 10 , was $26 \mathrm{~Gy}_{10}$ for whole brain and median $62.5 \mathrm{~Gy}_{10}$ (range, 39.4 to 71.0) for gross tumors, respectively. All targets and organs at risk were delineated on the fusion images of simulation computed tomography and diagnostic MRI. Gross tumor volume was defined as the gross intracranial lesion on T1-weighted enhancing axial images and clinical target volume as whole brain. Additional 3-5 mm margin was used to generate planning target volume for SIB on gross tumors and whole brain. Planning target volume for whole brain excluded HA zone which was defined as $5 \mathrm{~mm}$ volumetric expansion on both of hippocampus. Contouring of organs at risk including hippocampus was implemented as per the recommendation of RTOG 0933 trial contouring atlas (https:// www.rtog.org/CoreLab / ContouringAtlases / hippocampalsparing.aspx). Hippocampus was delineated as the hypointense area medial to the temporal horn on the T1-weighted axial MRIs. Dose constraint for hippocampus was as follows: dose at 100\% volume of hippocampus $(\mathrm{D} 100 \%) \leq 10 \mathrm{~Gy}$ and maximum dose to hippocampus $\leq 17 \mathrm{~Gy}$. Two patients were treated with ipsilateral hippocampal sparing due to the presence of metastatic tumor close $(<10 \mathrm{~mm})$ to contralateral hippocampus.

\section{Follow-up and statistical analysis}

A follow-up with brain MRI was scheduled for all patients 1-2 months after the finish of RT and every 3 months thereafter. Responses of metastatic brain tumors were evaluated by using the Response Evaluation Criteria in Solid Tumors [14]. A complete response (CR) was defined as the disappearance of the tumor; a partial response (PR) as a $30 \%$ reduction in the longest diameter of the tumor; progressive disease (PD) as a $\geq 20 \%$ increase in the longest diameter of the tumor, or the appearance of one or more new lesions; and a stable disease (SD) as a response that did not meet the condition of PR or PD. Local tumor progression was defined as a recurrence or tumor progression in SIB field, and intra-cranial progression as any recurrence or tumor progression within whole brain. Progression in HA zone was defined as the recurrence within the area expanded $5 \mathrm{~mm}$ from HA zone. Local tumor control, intracranial progression-free survival (PFS), and overall survival (OS) was calculated from the start date of RT to the date of local tumor progression, intracranial progression, and any death, respectively. Kaplan- Meier method was used to evaluate the survival rate. p-values of $<0.05$ were regarded as statistically significant.

\section{Ethical statement}

The review and analysis of the patient data was approved by Institutional Review Board (VC17RESI0189) of the St. Vincent's Hospital, the Republic of Korea. The informed consent was waived due to the retrospective design of the current study. 
Table 1. Patient and tumor characteristics $(n=42)$

\begin{tabular}{|c|c|}
\hline Parameter & No. $(\%)$ \\
\hline Age (yr) & $63(31-79)$ \\
\hline$\geq 60$ & $22(52.4)$ \\
\hline$<60$ & $20(47.6)$ \\
\hline \multicolumn{2}{|l|}{ Sex } \\
\hline Male & $30(71.4)$ \\
\hline Female & $12(28.6)$ \\
\hline \multicolumn{2}{|l|}{ Primary tumor site } \\
\hline Lung & $29(69.0)$ \\
\hline Rectum & $4(9.5)$ \\
\hline Kidney & $3(7.1)$ \\
\hline Breast & $2(4.8)$ \\
\hline Colon & $2(4.8)$ \\
\hline Ovary & $1(2.4)$ \\
\hline Thyroid & $1(2.4)$ \\
\hline \multicolumn{2}{|l|}{ Histology } \\
\hline Adenocarcinoma & $28(66.7)$ \\
\hline Non-adenocarcinoma & $14(33.3)$ \\
\hline \multicolumn{2}{|l|}{ KPS $(\%)$} \\
\hline$\geq 70$ & $35(83.3)$ \\
\hline$<70$ & $7(16.7)$ \\
\hline \multicolumn{2}{|l|}{ RPA class } \\
\hline I & $9(21.4)$ \\
\hline II & $26(61.9)$ \\
\hline III & $7(16.7)$ \\
\hline \multicolumn{2}{|l|}{ GPA score } \\
\hline $0-1.0$ & $12(28.6)$ \\
\hline $1.5-2.5$ & $27(64.3)$ \\
\hline $3.0-4.0$ & $3(7.1)$ \\
\hline No. of metastatic brain tumors & $3(1-12)$ \\
\hline 1 & $9(21.4)$ \\
\hline 2 & $12(28.6)$ \\
\hline 3 & $6(14.3)$ \\
\hline$\geq 4$ & $15(35.7)$ \\
\hline Maximum size of lesion in MRI (axial, cm) & $2.2(0.5-12.0)$ \\
\hline$\geq 3$ & $14(33.3)$ \\
\hline$<3$ & $28(66.7)$ \\
\hline \multicolumn{2}{|l|}{ Status of extracranial tumor } \\
\hline NED & $11(26.2)$ \\
\hline Stable & $6(14.3)$ \\
\hline Progressive & $11(26.2)$ \\
\hline Metastasis at diagnosis & $14(33.3)$ \\
\hline \multicolumn{2}{|l|}{ Treatment before WBRT } \\
\hline Yes & $9(21.4)$ \\
\hline Surgical resection & $5(11.9)$ \\
\hline Chemotherapy & $4(9.5)$ \\
\hline No & $33(78.6)$ \\
\hline
\end{tabular}

(Continued)
Table 1. Continued

\begin{tabular}{ll} 
Parameter & No. (\%) \\
Treatment after WBRT & \\
$\quad$ Yes & $30(71.4)$ \\
$\quad$ Target agent & $16(38.1)$ \\
No & $12(28.6)$ \\
\hline
\end{tabular}

KPS, Karnofsky performance status; RPA, recursive partitioning analysis; GPA, graded prognostic assessment; MRI, magnetic resonance imaging; NED, no evidence of disease; WBRT, whole brain radiotherapy.

\section{Results}

\section{Patient and tumor characteristics}

The characteristics of patient and tumor are summarized in Table 1. There were 30 men and 12 women, and the median age at HA-WBRT was 63 years (range, 31 to 79). Lung $(\mathrm{n}=29)$ was the most common primary site, followed by rectum $(n=4)$, kidney $(n=3)$, breast $(n=2)$, colon $(n=2)$, ovary $(n=1)$, and thyroid $(n=1)$. Adenocarcinoma was the most common histology $(28 / 42,66.7 \%)$. Thirty-five patients $(83.3 \%)$ had more than $70 \%$ of Karnofsky performance status. The recursive partitioning analysis (RPA) was distributed in nine patients (21.4\%) for class I, $26(61.9 \%)$ for II, and seven (16.7\%) for III. The Graded Prognostic Assessment [15] was scored with 0-1.0 in 12 patients (28.6\%), 1.5-2.5 in $27(64.3 \%)$, and 3.0-4.0 in $3(7.1 \%)$. The number of metastatic brain tumor was median 3 (range, 1 to 12 ). Twenty-seven patients (64.3\%) had 1-3 lesions, while $15(35.7 \%)$ indicated $\geq 4$ metastatic tumors. The median diameter of largest metastatic brain tumor was $2.2 \mathrm{~cm}$ (range, 0.5 to $12.0 \mathrm{~cm}$ ). There was no patient with leptomeningeal seeding at the time of HAWBRT. Seventeen patients $(40.5 \%)$ had stable or no evidence of disease for extracranial tumor, while $25(59.5 \%)$ had PD or metastasis at diagnosis. Nine patients $(21.4 \%)$ received preWBRT treatment, such as surgery or chemotherapy, for brain metastases. Of 30 patients $(71.4 \%)$ who received additional treatment after HA-WBRT, 16 (38.1\%: lung 10, kidney 3, rectum 2 , and colon 1 ) were treated with targeted agents.

Fig. 1 shows the dose distribution and dose-volume histogram of treatment plan for a patient treated with HA-WBRT as an example. A 40-year-old woman had two metastatic brain lesions from non-small cell lung cancer and received 25 Gy for whole brain and 45 Gy for brain metastases in 10 fractionations. 
A

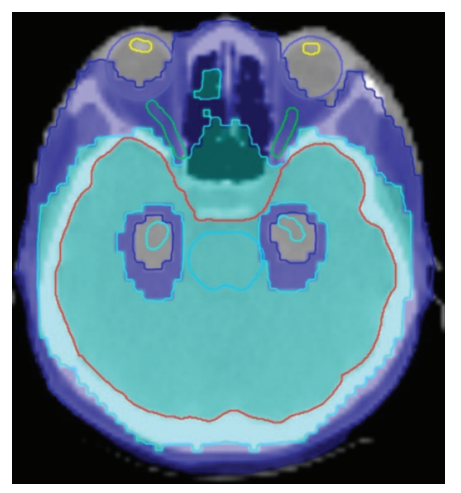

B

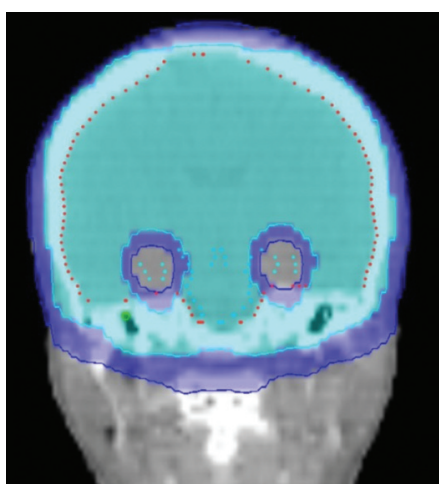

C

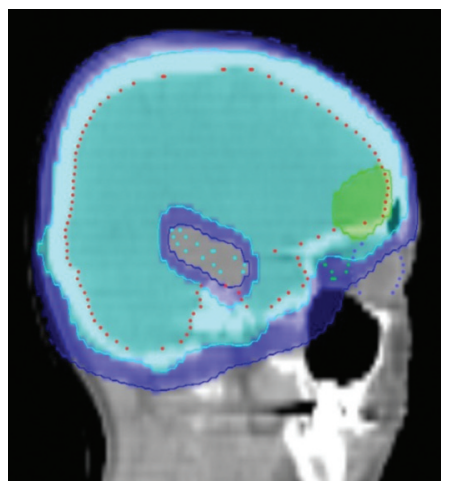

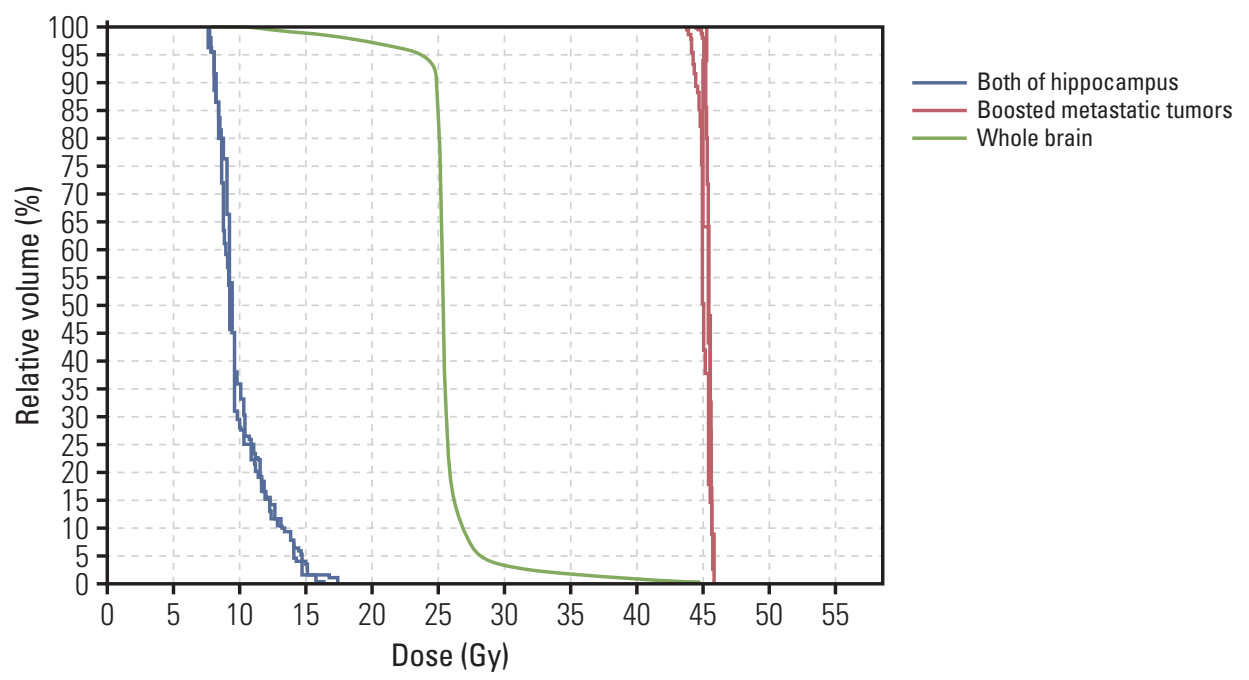

Fig. 1. Treatment plan of a 40-year-old woman who received whole brain radiotherapy with hippocampal avoidance for two brain metastases from non-small cell lung cancer. (A-C) Dose distribution of axial, coronal, and sagittal plane. (D) Dosevolume histogram for boosted metastatic tumors (red), whole brain (green), and both of hippocampus (blue).

\section{Survival outcome and patterns of failure}

Twenty-five patients (59.5\%) remained alive and $17(40.5 \%)$ died at the time of analysis. The median of follow-up and overall RT duration were 10.0 months (range, 4.1 to 56.4 months) and 13 days (range, 11 to 20 days), respectively. The response of intracranial metastatic lesion after HA-WBRT with SIB was CR in five patients (11.9\%), PR in 17 (40.5\%), SD in nine (21.4\%), and PD in $11(26.2 \%)$. The patterns of failure are described in Fig. 2. Intracranial progression was observed in 13 patients $(31.0 \%)$ and the median duration from the start of HA-WBRT to progression was 10.6 months (range, 0.9 to 33.0 months). Local tumor progression and new metastasis outside SIB field occurred in 10 patients (23.8\%) and nine patients $(21.4 \%)$, respectively. Progression in HA zone occurred only in one patient $(2.4 \%)$, who showed multiple metastases outside HA zone simultaneously. The 1-year rate of local tumor control was $76.6 \%$ (Fig. 3A). Median survival and 1-year rate for intracranial PFS were 19.4 months (95\% confidence interval [CI], 9.6 to 29.2 ) and $71.5 \%$ (Fig. 3B), and those for OS were 26.5 months (95\% CI, 15.4 to 37.5) and $67.9 \%$ (Fig. 3C).

Of 29 patients with primary origin of lung cancer, 10 patients showed intracranial progression. Post-WBRT targeted therapy was given to 10 patients, and two of them developed intracranial progression. There was no association between 


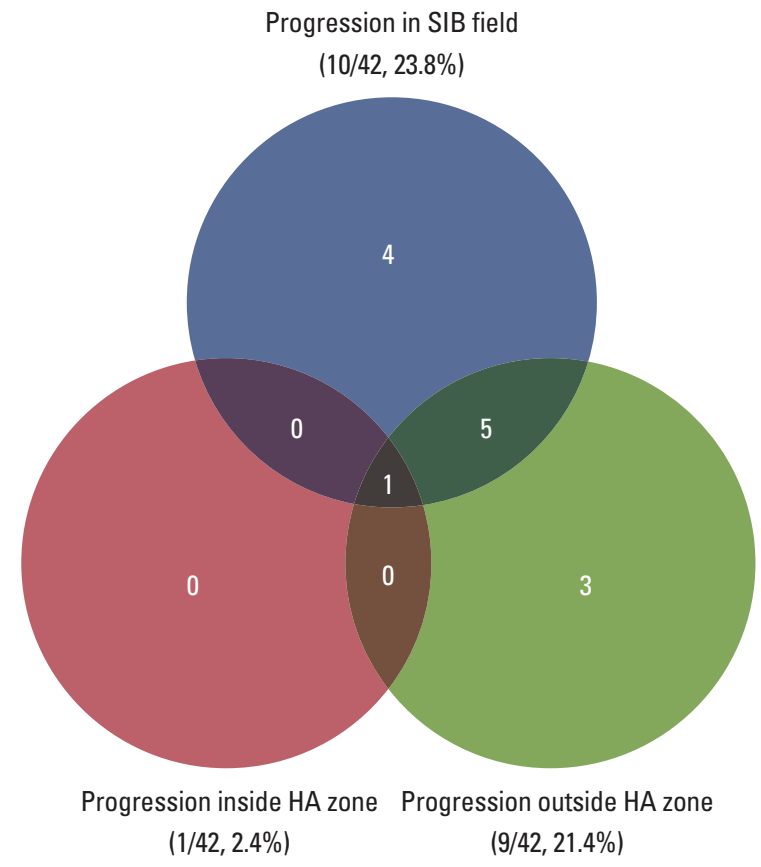

Fig. 2. Patterns of failure in patients who received whole brain radiotherapy with hippocampal avoidance for brain metastases. SIB, simultaneous integrated boost; HA, hippocampus avoidance.

targeted therapy and intracranial progression (target agent $2 / 10$ vs. non-target agent $8 / 19, p=0.414$ by Fisher exact test).

\section{Toxicity}

Of 42 patients, 11 were asked to answer the questions regarding their neurocognitive function. Three patients mentioned some deterioration in memory, but their alleged conditions were not assessed with an objective tool. Another one showed dysarthria for 3 months after treatment. There were no grade $\geq 3$ acute and late toxicities according to Common Terminology Criteria for Adverse Events, v4.03. Mild symptoms, such as headache, nausea, somnolence, fatigue, and dizziness were relieved with conservative managements. No patient had treatment break due to RT-related toxicities.

\section{Discussion}

According to a recent national cohort investigation [16], median survival duration and 1-year survival rate was $5.0 \pm 0.4$ months and $30.8 \pm 1.7 \%$ for patients with intracranial metastases, respectively. Despite well-known dismal prognosis, longer survival was anticipated for some patients with brain metastases and our results presented that 20 patients $(47.6 \%)$ had survived more than 12 months after WBRT with SIB on metastatic tumors. The studies that evaluated the effect of WBRT followed by radiosurgery boost indicate that intracranial tumor control could be improved and the survival prolonged in subgroup patients, although there is no significant increase for OS in whole patients $[17,18]$. Given that the favorable prognosis is shown in the subgroup patients with brain metastases, consideration should be given to the quality of life for those who received cranial irradiation. Other areas of interest included the early deterioration, less than 6 months, of recall and delayed recall and patient-reported quality of life observed in the patients with WBRT $[5,8-9,19]$.

In a phase II multi-institutional trial (RTOG 0933), Gondi et al. [20] demonstrated that the avoiding hippocampus during WBRT was associated with the preservation of memory and quality of life. The low risk of recurrence around hippocampus would be prerequisite for HA-WBRT. Several studies evaluated the incidence and elevated risk of excluding the hippocampus from irradiated volume for brain metastases. In the safety profile study for RTOG 0933, Gondi et al. [13] showed low incidence of brain metastases within HA region, defined as the hippocampus plus $5 \mathrm{~mm}$, through review of 1,133 metastatic lesion from 371 patients: $3 \%$ of brain metastases and $8.6 \%$ of patients. According to Harth et al. [21] who estimated the intracranial failure risk following HA-WBRT in the patients with non-small cell lung cancer, there was low incidence of $2.8 \%$ for brain metastasis in the hippocampus and there would be slightly increased absolute risk of $0.2 \%$ in case of delivering HA-WBRT. Sun et al. [22] recently investigated the incidence and relapse risk of perihippocampal metastases (around $5 \mathrm{~mm}$ the hippocampus) in patients with breast cancer. The reported rates of brain metastases in hippocampus were $1.2 \%$ of 1,678 metastases and $4.1 \%$ of 314 patients, and those of perihippocampal region were $3.5 \%$ and $11.1 \%$, respectively. They also presented the low absolute risk of $2.2 \%$ for the increase of perihippocamapal recurrence after HA-WBRT. According to another study that analyzed the incidence of melanoma metastasis to brain, none $(0.0 \%)$ and four of 77 patients $(5.2 \%)$ had metastases within and within $5 \mathrm{~mm}$ of the hippocampus, respectively [23]. Consistent with the previous studies that presented low incidences of hippocampal metastasis, our study shows that only one patient $(2.4 \%)$ experienced new metastasis in hippocampal region following HA-WBRT, confirming the safety of this hypothetical radiation delivery strategy. Besides, due consideration should be given to the fact that the patient also showed miliary spread in the whole brain as well as hippocampal metastasis. This finding might 
A
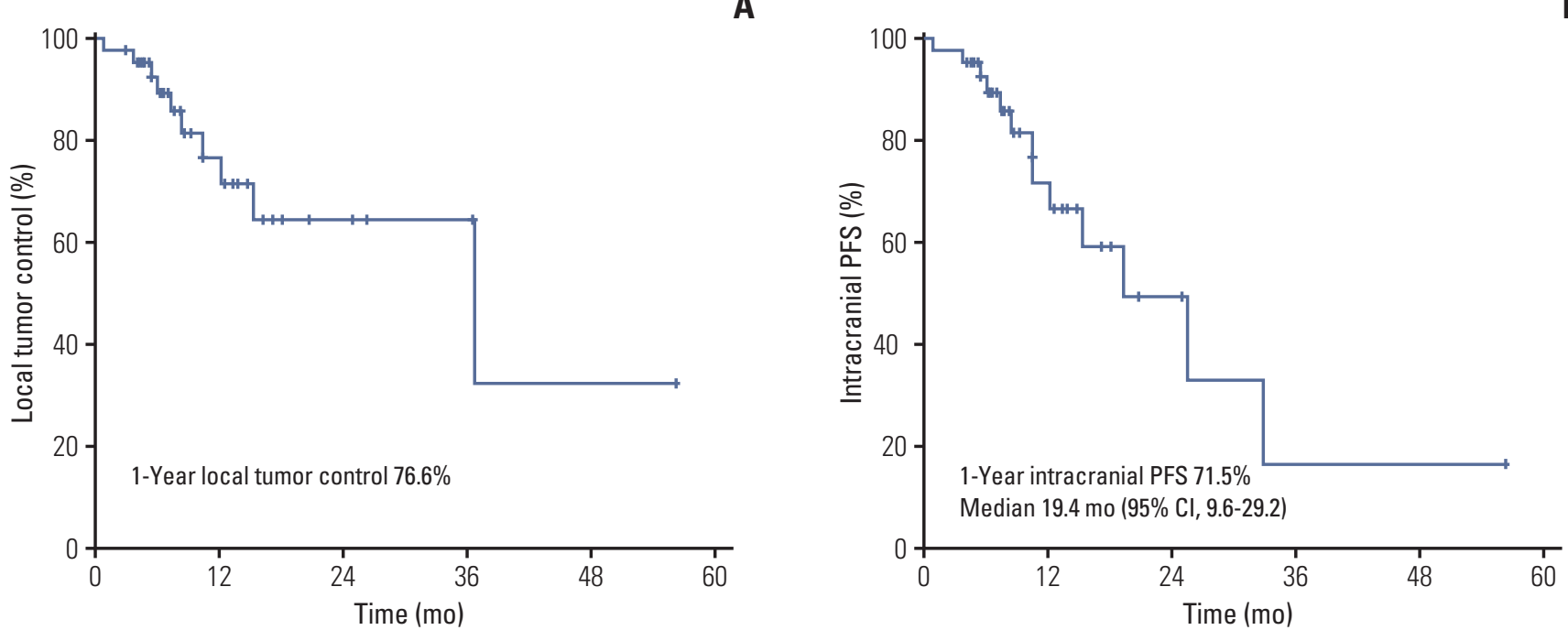

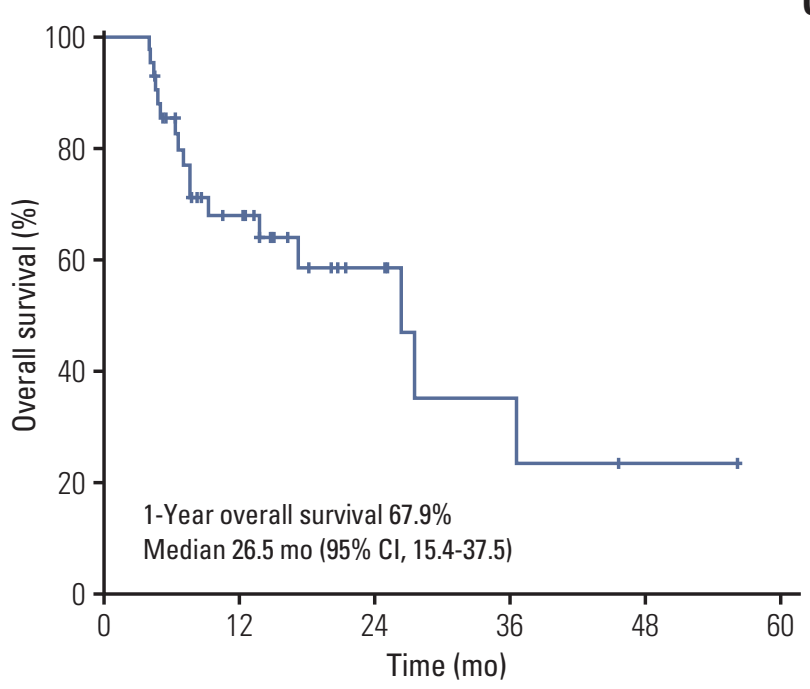

Fig. 3. Local tumor control (A), intracranial progression free survival (PFS) (B), and overall survival (C) in patients who received whole brain radiotherapy with hippocampal avoidance for brain metastases. CI, confidence interval.

imply that the prognosis of the patient would not have been changed even if he had received WBRT without hippocampal sparing.

Oehlke et al. [24] reported the favorable local tumor control with acceptable HA zone metastasis in 20 patients who received WBRT with hippocampal sparing for multiple brain metastases. Cranial irradiation with hippocampal sparing was performed on whole brain with $30 \mathrm{~Gy}$ in 12 fractions (EQD2, $31.3 \mathrm{~Gy}_{10}$ ) and SIB on metastatic tumors with $51 \mathrm{~Gy}$ in 12 fractions (EQD2, $60.6 \mathrm{~Gy} 10$ ) using volumetric modulated arc therapy approach. There were two patients who devel- 
patients (27/42, 64.3\%) had 1-3 metastatic lesion (median, 3; range, 1 to 12) in our study, while all the patients had brain metastases of median 5 (range, 2 to 13) in Oehlke et al.'s [24]. The proportion of patients with RPA class I and post-WBRT treatment might be other factors that have influenced on intracranial control and survival.

A total of 16 patients were treated with post-WBRT targeted therapy (lung 10, kidney 3 , rectum 2 , and colon 1 ) in our study. To confine the origin of lung cancer, two patients developed intracranial progression and eight didn't. There was no statistically significant difference for incidence $(p=0.414)$, but relatively prolonged time to progression for intracranial recurrence. One patient had intracranial PFS of 33.0 months, while the other only 8.5 months who had stopped the gefitinib 3 months before developing intracranial progression without her physician's permission. Compared with median survival of 15.9-21.9 months in the patients who received erlotinib or gefitinib for brain metastases from lung cancer [25-27], 33.0 months of intracranial PFS would be noteworthy for the efficacy of WBRT in addition to targeted therapy.

Our study has some limitations due to small retrospective nature. First, it could be underestimated for intracranial PFS in some patients due to inconsistent follow-up of post-WBRT MRI. Second, there would be confounding factors for local tumor control and survival, because of a heterogeneous group of patients and disease status. Third, the patients in our study were not assessed on impaired function of hippocampus by objective tools, such as Hopkins Verbal Learning Test-Revised. Fewer than a third of patients $(11 / 42$, $26.2 \%$ ) were asked to have their memory status evaluated before and after WBRT by an attending physician and dropouts were a problem in evaluating neurocognitive function.

In conclusion, the current study confirmed an acceptable low risk of new metastasis in HA region in the patients with brain metastases who received HA-WBRT. These findings would serve as useful guidance in applying HA-WBRT in clinical practice. Further investigation is warranted in a larger sampled multi-institutional study.

\section{Conflicts of Interest}

Conflict of interest relevant to this article was not reported.

\section{References}

1. Khuntia D, Brown P, Li J, Mehta MP. Whole-brain radiotherapy in the management of brain metastasis. J Clin Oncol. 2006;24:1295-304.

2. Diener-West M, Dobbins TW, Phillips TL, Nelson DF. Identification of an optimal subgroup for treatment evaluation of patients with brain metastases using RTOG study 7916. Int J Radiat Oncol Biol Phys. 1989;16:669-73.

3. Gaspar LE, Mehta MP, Patchell RA, Burri SH, Robinson PD, Morris RE, et al. The role of whole brain radiation therapy in the management of newly diagnosed brain metastases: a systematic review and evidence-based clinical practice guideline. J Neurooncol. 2010;96:17-32.

4. Tsao MN, Rades D, Wirth A, Lo SS, Danielson BL, Gaspar LE, et al. Radiotherapeutic and surgical management for newly diagnosed brain metastasis(es): an American Society for Radiation Oncology evidence-based guideline. Pract Radiat Oncol. 2012;2:210-25.

5. Chang EL, Wefel JS, Hess KR, Allen PK, Lang FF, Kornguth DG, et al. Neurocognition in patients with brain metastases treated with radiosurgery or radiosurgery plus whole-brain irradiation: a randomised controlled trial. Lancet Oncol. 2009;10:1037-44.

6. Patchell RA. The management of brain metastases. Cancer Treat Rev. 2003;29:533-40.

7. Patchell RA, Tibbs PA, Regine WF, Dempsey RJ, Mohiuddin $\mathrm{M}$, Kryscio RJ, et al. Postoperative radiotherapy in the treat- ment of single metastases to the brain: a randomized trial. JAMA. 1998;280:1485-9.

8. Soffietti R, Kocher M, Abacioglu UM, Villa S, Fauchon F, Baumert BG, et al. A European Organisation for Research and Treatment of Cancer phase III trial of adjuvant whole-brain radiotherapy versus observation in patients with one to three brain metastases from solid tumors after surgical resection or radiosurgery: quality-of-life results. J Clin Oncol. 2013;31:6572.

9. Welzel G, Fleckenstein K, Schaefer J, Hermann B, KrausTiefenbacher U, Mai SK, et al. Memory function before and after whole brain radiotherapy in patients with and without brain metastases. Int J Radiat Oncol Biol Phys. 2008;72:1311-8.

10. Mizumatsu S, Monje ML, Morhardt DR, Rola R, Palmer TD, Fike JR. Extreme sensitivity of adult neurogenesis to low doses of X-irradiation. Cancer Res. 2003;63:4021-7.

11. Monje ML, Mizumatsu S, Fike JR, Palmer TD. Irradiation induces neural precursor-cell dysfunction. Nat Med. 2002;8: 955-62.

12. Nagai R, Tsunoda S, Hori Y, Asada H. Selective vulnerability to radiation in the hippocampal dentate granule cells. Surg Neurol. 2000;53:503-6.

13. Gondi V, Tome WA, Marsh J, Struck A, Ghia A, Turian JV, et al. Estimated risk of perihippocampal disease progression after hippocampal avoidance during whole-brain radiotherapy: safety profile for RTOG 0933. Radiother Oncol. 2010;95: 
327-31.

14. Eisenhauer EA, Therasse P, Bogaerts J, Schwartz LH, Sargent D, Ford R, et al. New response evaluation criteria in solid tumours: revised RECIST guideline (version 1.1). Eur J Cancer. 2009;45:228-47.

15. Sperduto PW, Berkey B, Gaspar LE, Mehta M, Curran W. A new prognostic index and comparison to three other indices for patients with brain metastases: an analysis of 1,960 patients in the RTOG database. Int J Radiat Oncol Biol Phys. 2008;70: 510-4.

16. Kim T, Song C, Han JH, Kim IA, Kim YJ, Kim SH, et al. Epidemiology of intracranial metastases in Korea: a national cohort investigation. Cancer Res Treat. 2018;50:164-74.

17. Andrews DW, Scott CB, Sperduto PW, Flanders AE, Gaspar $\mathrm{LE}$, Schell MC, et al. Whole brain radiation therapy with or without stereotactic radiosurgery boost for patients with one to three brain metastases: phase III results of the RTOG 9508 randomised trial. Lancet. 2004;363:1665-72.

18. Kondziolka D, Patel A, Lunsford LD, Kassam A, Flickinger JC. Stereotactic radiosurgery plus whole brain radiotherapy versus radiotherapy alone for patients with multiple brain metastases. Int J Radiat Oncol Biol Phys. 1999;45:427-34.

19. Li J, Bentzen SM, Renschler M, Mehta MP. Regression after whole-brain radiation therapy for brain metastases correlates with survival and improved neurocognitive function. J Clin Oncol. 2007;25:1260-6.

20. Gondi V, Pugh SL, Tome WA, Caine C, Corn B, Kanner A, et al. Preservation of memory with conformal avoidance of the hippocampal neural stem-cell compartment during wholebrain radiotherapy for brain metastases (RTOG 0933): a phase II multi-institutional trial. J Clin Oncol. 2014;32:3810-6.
21. Harth S, Abo-Madyan Y, Zheng L, Siebenlist K, Herskind C, Wenz $\mathrm{F}$, et al. Estimation of intracranial failure risk following hippocampal-sparing whole brain radiotherapy. Radiother Oncol. 2013;109:152-8.

22. Sun B, Huang Z, Wu S, Shen G, Cha L, Meng X, et al. Incidence and relapse risk of intracranial metastases within the perihippocampal region in 314 patients with breast cancer. Radiother Oncol. 2016;118:181-6.

23. Hong AM, Suo C, Valenzuela M, Haydu LE, Jacobsen KD, Reisse $\mathrm{CH}$, et al. Low incidence of melanoma brain metastasis in the hippocampus. Radiother Oncol. 2014;111:59-62.

24. Oehlke O, Wucherpfennig D, Fels F, Frings L, Egger K, Weyerbrock $\mathrm{A}$, et al. Whole brain irradiation with hippocampal sparing and dose escalation on multiple brain metastases: Local tumour control and survival. Strahlenther Onkol. 2015;191: 461-9.

25. Iuchi T, Shingyoji M, Sakaida T, Hatano K, Nagano O, Itakura $\mathrm{M}$, et al. Phase II trial of gefitinib alone without radiation therapy for Japanese patients with brain metastases from EGFRmutant lung adenocarcinoma. Lung Cancer. 2013;82:282-7.

26. Kim JE, Lee DH, Choi Y, Yoon DH, Kim SW, Suh C, et al. Epidermal growth factor receptor tyrosine kinase inhibitors as a first-line therapy for never-smokers with adenocarcinoma of the lung having asymptomatic synchronous brain metastasis. Lung Cancer. 2009;65:351-4.

27. Park SJ, Kim HT, Lee DH, Kim KP, Kim SW, Suh C, et al. Efficacy of epidermal growth factor receptor tyrosine kinase inhibitors for brain metastasis in non-small cell lung cancer patients harboring either exon 19 or 21 mutation. Lung Cancer. 2012;77:556-60. 